\title{
Probabilistic production simulation of a power system with wind power penetration based on improved UGF techniques
}

\author{
Hongtao WANG (ه), Xu LIU, Chunyi WANG
}

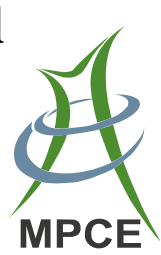

\begin{abstract}
Universal Generating Function (UGF) techniques have been applied to Multi-State System (MSS) reliability analysis, such as long term reserve expansion of power systems with high wind power penetration. However, using simple steady-state distribution models for wind power and large generating units in reliability assessment can yield pessimistic appraisals. To more accurately assess the power system reliability, UGF techniques are extended to dynamic probabilistic simulation analysis on two aspects of modelling improvement. Firstly, a principal component analysis (PCA) combined with a hierarchal clustering algorithm is used to achieve the salient and time-varying patterns of wind power, then a sequential UGF equivalent model of wind power output is established by an apportioning method. Secondly, other than the traditional two-state models, the conventional generator UGF equivalent model is established as a four discrete-state continuous-time Markov model by $L z$ transform. In the construction process of such a UGF model, the state values are transformed into the integral multiples of one common factor by choosing proper common factors, thus effectively restraining the exponential growth of its state number and alleviating the explosion thereof. The method is suitable for reliability assessment with dynamic probabilistic distributed random
\end{abstract}

Received: 5 February 2013/ Accepted: 1 June 2013/Published online: 12 September 2013

(C) The Author(s) 2013. This article is published with open access at Springerlink.com

H. WANG, Key Laboratory of Power System Intelligent

Dispatch and Control of Ministry of Education, Shandong

University, Jinan 250061, China

(ه) e-mail: whtwhm@sdu.edu.cn

X. LIU, State Grid Zhejiang Electric Power Company, Jiaxing

Power Supply Company, Jiaxing 314001, China

C. WANG, State Grid Shandong Electric Power Company,

Jinan 250001, China variables. In addition, by acquiring the clustering information of wind power, the system reliability indices, such as fuel cost and $\mathrm{CO}_{2}$ emissions through different seasons and on different workdays, are calculated. Finally, the effectiveness of the method is verified by a modified IEEE-RTS 79 system integrated with several wind farms of historical hourly wind power data of Zhangbei wind farm in North China.

Keywords Multi-state system, Reliability, Universal Generating Function (UGF), Probabilistic production simulation, Wind power

\section{Introduction}

Compared with Monte Carlo simulation techniques [14] and analytical methods [5-8], an Universal Generating Function (UGF) technique $[9,10]$ is the most promising analysis tool for the probabilistic production simulation of power systems, and has been widely used in the probabilistic production simulation analysis of power systems.

However, the main restriction of this powerful technique is that, theoretically it can only deal with random variables, and can only obtain the steady-state performance distributions of wind power and conventional generators. By using a basic UGF technique, it is impossible to analyze the dynamic probability distribution modes of reliability systems, including component aging reliability models, and non-stationary stochastic process of wind power under diurnal and seasonal pattern features, etc. Besides, there has been a number explosion in states of large systems. In this paper, two aspects of UGF modelling improvement are presented. On UGF modelling aspect of wind power, a hierarchal clustering method for constructing the timevariance UGF is proposed according to the non-stationary stochastic characteristics of wind power. Firstly, principal 
component analysis (PCA) [11] combined with a hierarchal clustering algorithm [12] is adopted to obtain the typical time-sequential samples of wind power output. An apportioning method [13] is used to establish the sequential UGF of the wind power output.

Besides the wind power output, the outputs of conventional generating units are not uniformly distributed too. Reference [14] proposed a multi-state Markov model for a coal-fired power generating unit, and a technique for the estimation of transition intensities between the various generating capacity levels of the unit based on the actual measurements. The technique can be used to estimate the dynamic distributions of generating capacity distribution. Based on the technique, the UGF of the conventional generating unit is formulated as a sequential UGF with an $L z$-transform, which can express the time-varying multistate Markov process as an UGF equivalent model [15]. The sequential UGF models for conventional generators reflect the impact of time-varying factors in operation and repair after failures. As a result, each component in power systems is formulated as a sequential UGF model, which will extend the UGF technique applicability to dynamic probabilistic production simulation analysis.

Furthermore, state values expressed as a multiple of the common factor, can avoid the exponential growth of the state combination number, thus the problem of number explosion of states is avoided in the proposed method. Finally, the proposed approach presents the ability to quantify the impact of the temporal correlation between wind power and load on the assessment of system reliability indices, fuel cost and $\mathrm{CO}_{2}$ emissions.

The rest of the paper is organized as follows. The UGF technique is briefly summarized in Section 2 . The reliability modelling of wind farms, conventional generating units, loads and system is presented with UGF technique in Section 3. The procedure of power system reliability assessment based on the dynamic probability distribution of the conventional generator and different patterns of wind farm or loads is proposed in Section 4. Case studies are provided in Section 5, followed by the conclusions in Section 6.

\section{UGF technique}

UGF technique is to find MSS reliability measures by using Ushakov's Universal generating Operator (UGO) $\Omega_{f}$ acting on the collection of the individual UGFs expression of all the individual random variables [10].

\subsection{UGF expression}

The basis of the UGF technique is to express the random variables as UGF expressions after $z$ transform. Supposing that there are two discrete random variables $X_{1}$ and $X_{2}$, the corresponding probability function is:

$$
\begin{cases}\operatorname{Pr}\left\{X_{1}=x_{1 i}\right\}=p_{1 i}, & 1 \leq i \leq k_{1} \\ \operatorname{Pr}\left\{X_{2}=x_{2 i}\right\}=p_{2 i}, & 1 \leq i \leq k_{2}\end{cases}
$$

where $x_{1 i}$ is the $i$-th state of $X_{1}$, and the corresponding probability is $p_{1 i} ; x_{2 i}$ is the $i$-th state of $X_{2}$, and the corresponding probability is $p_{2 i} ; k_{1}$ and $k_{2}$ are the state numbers of $X_{1}$ and $X_{2}$. After $z$ transform, the corresponding UGF becomes:

$$
\left\{\begin{array}{l}
z\left\{x_{1}\right\}=u_{x_{1}}(z)=\sum_{i=1}^{k_{1}} p_{1 i} z^{x_{1 i}}=p_{11} z^{x_{11}}+p_{12} z^{x_{12}}+\cdots+p_{1 k_{1}} z^{x_{1 k_{1}}} \\
z\left\{x_{2}\right\}=u_{x_{2}}(z)=\sum_{j=1}^{k_{2}} p_{2 j} z^{x_{2 j}}=p_{21} z^{x_{11}}+p_{22} z^{x_{22}}+\cdots+p_{2 k_{2}} z^{x_{2 k_{2}}}
\end{array}\right.
$$

where $u_{x_{1}}(z)$ and $u_{x_{2}}(z)$ are the UGFs of $X_{1}$ and $X_{2} ; z$ has no substantial meaning.

The UGF expression provides a way to distinguish the values of random variables from the corresponding probability with $z$ transform. The $z$ transform can be extended to the UGO $\Omega_{f}$ for a function that concludes random variables.

Supposing that $Y$ is an arbitrary function of $x_{1}$ and $x_{2}$, so $Y=f\left(x_{1}, x_{2}\right)$. The $z$ transform $u_{Y}(z)$ for $Y$ can be formally obtained as a product of individual $z$ transforms of $x_{1}$ and $x_{2}$ by the UGO $\Omega_{f}$ :

$$
\begin{aligned}
z\{Y\} & =u_{Y}(z)=\sum_{j=1}^{K} q_{j} z^{y_{j}}=\Omega_{f}\left\{u_{1}(z), u_{2}(z)\right\} \\
& =\sum_{j_{1}=1}^{k_{1}} \sum_{j_{2}=1}^{k_{2}} p_{1 j_{1}} p_{2 j_{2}} z^{f\left(x_{1 j_{1}}, x_{2 j_{2}}\right)}
\end{aligned}
$$

where $q_{j}=\left\{q_{1}, q_{2}, \ldots, q_{K}\right\}$ and $y_{j}=\left\{y_{j 1}, y_{j 2}, \ldots, y_{j K}\right\}$ are the resulting probability mass function and the $i$-th state of random variable $Y$, respectively.

\subsection{Sequential UGFs for time-varying process}

For a function $Y(t)=f\left(x_{1}(t), x_{2}(t), \ldots, x_{m}(t)\right)$, which concludes discrete-state continuous-time random variables $x_{1}(t), x_{2}(t), \ldots, x_{m}(t), z$ transform is extended to $L z$-transform as [15]:

$$
\begin{aligned}
& L_{z}\{Y(t)\}=u_{Y}\left(z, t, p_{0}\right)=\sum_{j=1}^{K} q_{j}(t) z^{y_{j}(t)} \\
& \quad=\Omega_{f}\left\{u_{x 1}(z, t), u_{x 2}(z, t), \ldots, u_{x m}(z, t)\right\} \\
& \quad=\sum_{j_{m}}^{k_{m}} \cdots \sum_{j_{2}=1}^{k_{2}} \sum_{j_{1}=1}^{k_{1}} p_{1 j_{1}}(t) p_{2 j_{2}}(t) \cdots p_{m j_{m}}(t) z^{f\left(x_{j_{1}}(t), x_{2 j_{2}}(t), \ldots, x_{m j_{m}}(t)\right)}
\end{aligned}
$$


where $p_{1 i}(t), p_{2 i}(t), \ldots, p_{m i}(t)$ are the probability of $x_{1}(t), x_{2}(t), \ldots, x_{m}(t)$ at period $t$ for any given initial states probability $p_{0}$.

Straightforward computation of the probability mass function (p.m.f.) of $Y(t)$ using (4) is based on an enumerative approach. This approach is extremely resource consuming. Fortunately, there are two effective ways to reduce the computational burden: similar-terms collection and a recursive procedure [10].

The UGFs inherit the essential property of regular polynomials: they allow for collecting similar terms. If a UGF representing the p.m.f. of a random variable $X$ contains the terms $p_{h} z^{x_{h}}$ and $p_{m} z^{x_{m}}$ for which $x_{h}=x_{m}$, the two terms can be replaced by a single term $\left(p_{h}+p_{m}\right) z^{x_{m}}$. Due to the feature, a reasonable common capacity factor $\Delta c$ is chosen; and then, all of the state values, including wind generators, conventional generators and loads, are chosen as integer multiples of $\Delta c$; finally, the identical states are merging. Therefore, the number of states is prevented from increasing exponentially with the improved UGF technique for the reliability assessment of power systems.

\section{Reliability modelling with UGF technique}

\subsection{Reliability model for wind farms}

\subsubsection{Feature extraction technology of wind power and clustering algorithm}

The transformation of wind speed to wind power involves a cubic relationship, if there is an error in the construction of wind speed model, the error in the construction of wind power model will be three times greater [16]. And then the wind power output model of wind farms is established by using wind power data. By a principal component feature extraction technology [11] and a hierarchical clustering algorithm [12], it shows that the representative samples of wind power from historical time series, and then the UGF equivalent of wind farms is expressed as time variant according to the patterns after clustering.

According to [12], a day can be taken as a clustering time unit. Because of inhomogeneities in the measurement data, this hierarchical clustering algorithm combined with PCA feature extraction technology is applied to achieving the patterns of wind power. PCA can be used to identify the patterns from the data, and to express the data in a way that highlights their similarities and differences. PCA is a useful tool for dealing with large data sets, in which extracting the features becomes an important step.
PCA method is suitable for feature extraction of wind power output. Supposing that there are $p$ variables in the time series of wind power: $x_{1}, x_{2}, \ldots, x_{p}$. A $p$-dimensional random vector $x$ constituted by $p$ variables as $x=\left(x_{1}, x_{2}, \ldots, x_{p}\right)$. Supposing $x$ can be linearizedly transformed as follows:

$\left\{\begin{array}{c}y_{1}=\mu_{11} x_{1}+\mu_{12} x_{2}+\mu_{1 p} x_{p} \\ y_{2}=\mu_{21} x_{1}+\mu_{22} x_{2}+\mu_{2 p} x_{p} \\ \vdots \\ y_{p}=\mu_{p 1} x_{1}+\mu_{p 2} x_{2}+\mu_{p p} x_{p}\end{array}\right.$

where $y_{1}, y_{2}, \ldots, y_{p}$ are linear combinations of $x_{1}, x_{2}, \ldots, x_{p}$. In (5), every vector $\mu_{i}=\left(\mu_{i 1}, \mu_{i 2}, \ldots, \mu_{i p}\right)$ is a unit vector. And $y_{1}$ has the largest variance of linear combinations of $x_{1}, x_{2}, \ldots, x_{p} ; y_{2}$ exhibits the largest variance of linear combinations unrelated to $y_{1} ; y_{p}$ has the largest variance of linear combinations unrelated to $y_{1}, y_{2}, \ldots, y_{p-1}$. So $y_{1}, y_{2}, \ldots, y_{p}$ are called the first, second, ..,p $p$-th main components of the original variables.

According to the significances of the main components, the weaker component (i.e., those with smaller variance) can be removed. Supposing that we obtain $q(q \leq p)$ main components, i.e., $q$ orthogonal variables, so the original wind power output is mapped into a much smaller space, thus resulting in a concomitant dimension reduction. The value of main component $q$ is determined by cumulative contribution rate. If the cumulative contribution rate exceeds $85 \%$, the information in the original variables can be sufficiently reflected, and the corresponding $q$ refers to the main components extracted in $q$. Firstly, the wind power data are clustering after feature extraction and each object forms a separate group. Secondly, similar groups are merging until only one group is left or the termination conditions are met. The similarity, which is the basis of similar measurement clustering, is performed by using the Euclidean distance:

$d_{i j}=\sqrt{\sum_{k=1}^{q}\left(P_{w i}(k)-P_{w j}(k)\right)^{2}}$

where $P_{w i}$ and $P_{w j}$ are wind power vectors containing $q$ main components.

Let $D$ be the number of days studied, and $R_{w}$ be the number of wind power output patterns, denoted by $1,2, \ldots$, $r_{w}, \ldots, R_{w}$. The number of wind power outputs of pattern $r_{w}$ is $N_{r w}$, so the probability of pattern $r_{w}$ is $\alpha_{r_{w}}=N_{r_{w}} / D$.

The $R_{w}$ wind power patterns is obtained using a hierarchical clustering algorithm combined with principal component-feature extraction technology with probability $\alpha_{r_{w}}$, denoted by $\left(r_{w}, \alpha_{r_{w}}\right)\left(r_{w} \in\left\{1,2, \ldots, R_{w}\right\}\right)$.

\subsubsection{UGF equivalent of wind farm}

Each pattern of wind power is partitioned into $H$ subperiods, and an hourly resolution $(H=24)$ is adopted. 
Modelling UGF for wind power in different sub-periods with apportioning method [13], supposing that all wind power output curves of wind power pattern $r_{w}$ are shown in Fig. 1, and the number of curves is $N_{r_{w}}$, so each interval has $N_{r_{w}}$ wind power values. We can obtain state values and the corresponding probabilities of each interval using the apportioning method. Supposing that the $i$-th wind farm has $k_{w}$ states in pattern $r_{w}$ in sub-period $t$, the UGF becomes:

$u_{i}^{w}(z, t)=\sum_{j_{w}=1}^{k_{w}} p_{i, j_{w}}^{w}(t) z^{W P_{i, j_{w}}(t)}$

where $W P_{i, j_{w}}(t)$ and $p_{i, j_{w}}^{w}(t)$ are wind power output and the corresponding probability of the $i$-th wind farm in state $j_{w}$, respectively.

From (7), it can be seen that the UGF equation of wind power is expressed as timing variations form, so the improved UGF can reflect the dynamic probabilistic distribution of wind power.

\subsection{Reliability model for conventional generation units}

A reliability model for the traditional conventional generators is established according to historical statistical data, but in actual systems, the failure rate is time-varying. The reliability model of the conventional generation unit is presented as a discrete-state continuous-time Markov process in this paper. Supposing that the states of the $i$-th conventional generator can be represented as the following polynomial [14]:

$P_{i}^{m}(t)=\left\langle p_{i, j_{m}}^{m}, A_{i}^{m}, p_{0}^{m}\right\rangle$

where $p_{i, j_{m}}^{m}$ is the probability of a conventional generator $i$ in state $j_{m}, A_{i}^{m}$ is the transition probability intensities matrix, and $p_{0}^{m}$ is the initial state probability vector of the conventional generator $i$.

$L z$-transform of a discrete-state continuous-time Markov process of (8) is a UGF defined as [15]:

$L z\left\{P_{i}^{m}(t)\right\}=u_{i}^{m}\left(z, t, p_{0}\right)=\sum_{j_{m}=1}^{k_{m}} p_{i, j_{m}}^{m}(t) z^{P_{i, j m}(t)}$

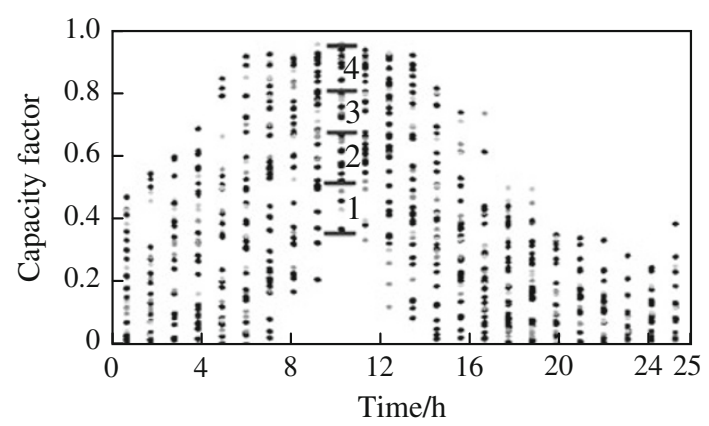

Fig. 1 Four-state wind power in pattern $r_{w}$ where $P_{i, j_{m}}(t)$ and $p_{i, j_{m}}^{m}(t)$ are the available power of conventional generator $i$ in state $j_{m}$ and the corresponding probability at sub-period $t$ for given probability distribution of initial states $p_{0}$. The total state number of the generator $i$ is $k_{m}$. Four-state Markov model is used for the units. Considering that a unit has four states $1,2,3$, and 4 with corresponding performance level $P_{1}=0, P_{4}=P_{n o m}$ and the values of $P_{2}, P_{3}$ can be obtained by the apportioning method [13], $p_{j_{m}}(t)$ is a probability and the process is in state $j_{m}$ at time instant $t \geq 0$. The transition intensity matrix $A_{m_{i}}^{m}=\left(a_{j_{m} q_{m}}\right)\left(j_{m}, q_{m}=1,2, \ldots, 4\right)$ can be obtained as the following polynomial [14]:

$$
\begin{aligned}
& T_{\sum j_{m}}=\sum_{m=1}^{k_{j_{m}}} T_{j_{m}}^{(m)} \\
& a_{j_{m} q_{m}}=\frac{k_{j_{m} q_{m}}}{T_{\sum j_{m}}}, \quad j_{m} \neq q_{m} \\
& a_{j_{m} j_{m}}=-\sum_{\substack{q_{m}=1 \\
q_{m} \neq j_{m}}}^{N} a_{j_{m} q_{m}}+1
\end{aligned}
$$

where $k_{j_{m}}$ is the accumulated number of the unit residence in state $j_{m}$ during observation period $T, T_{j_{m}}^{(m)}$ is the time of the $m$-th unit residence in state $j_{m}$ during observation period $T, T_{\sum j_{m}}$ is the accumulated time of the system residence in each state $j_{m}$ during observation period $T, k_{j_{m} q_{m}}$ is the accumulated number of the unit transition from state $j_{m}$ to state $q_{m}$ during observation period $T$.

With the differential equations in [14], state-probabilities $p_{j_{m}}(t)$ can be obtained under given initial conditions. Therefore, dynamic reliability measures for generating units can also be obtained.

\subsection{Reliability model for load model}

The historical data of load, such as wind power data, are handled by using a hierarchical clustering algorithm and principal component feature extraction technology. The UGF model of load $i$ in pattern $r_{L}$ at sub-period $t$ is described as follows:

$u_{i}^{L}(z, t)=\sum_{j_{L}=1}^{k_{L}} p_{i, j_{L}}^{L}(t) z^{D_{i, j_{L}}(t)}$

where $D_{i, j_{L}}(t)$ and $p_{i, j_{L}}^{L}(t)$ are the load capacity and probability, respectively, $k_{L}$ is the total state number of loads.

\subsection{System UGF considering transmission network}

System UGF combines UGFs of all power system components, including UGF of transmission network 
which is formulated as system states. For a $N$-bus system with $k_{n}$ system states, the system UGF can be obtained by the optimal power flow operator $\Omega_{\Phi O P F}$ :

$$
\begin{aligned}
u^{s}(z, t)= & \Omega_{\text {ФOPF }}\left\{u_{i}^{L}(z, t), u_{i}^{w}(z, t), u_{i}^{m}(z, t)\right\} \\
= & \sum_{i=1}^{N} \sum_{j_{L}=1}^{k_{L}} \sum_{j_{w}=1}^{k_{w}} \sum_{j_{m}=1}^{k_{m}} \sum_{j_{n}=1}^{k_{n}} p_{i, j_{L}}^{L}(t) \cdot p_{i, j_{w}}^{w}(t) \\
& \cdot p_{i, j_{m}}^{m}(t) \cdot p_{j_{n}}^{T}(t) \\
& \cdot z^{\Phi_{O P F}}\left\{D_{i, j_{L}}(t), W P_{i, j_{w}}(t), P_{i, j_{m}}(t)\right\} \\
= & \sum_{j_{s}=1}^{K_{s}} p_{j_{s}}^{s}(t) z^{L C_{j_{s}}(t)}
\end{aligned}
$$

where $p_{j_{s}}^{s}(t)$ and $L C_{j_{s}}(t)$ are the probability and load curtailment of system state $j_{s}$, respectively, $p_{j_{n}}^{T}(t)$ is the probability for the transmission network state $j_{n}, K_{s}$ is the total state number including all state combination.

\section{Probabilistic production simulation}

On the basis of the probabilistic production simulation method [17], and the improved UGF techniques, considering the minimum outage time constraints and peak-shaving constraints of generator, a UGF model can be established. During the probabilistic production simulation of power systems, wind power, thermal generator and peak-shaving units are dispatched. In UGF, conventional units are divided into several levels of output, which have different generating costs per MWh according to base load (minimum output part) and peak load (adjustable output part) for load dispatching.

After simulation, we can obtain the reliability index loss of load probability $L_{\mathrm{LOLP}, r}(t)$ and Expected Energy Not Supplied $E_{\mathrm{EENS}, r}(t)$ in each composite pattern $r_{s}$ at sub-period $t$ :

$L_{\mathrm{LOLP}, r_{s}}(t)=1-p_{j_{s} \mid L C_{j_{s}}(t)=0}^{s}(t)$

$E_{\mathrm{EENS}, r_{s}}(t)=\sum_{j_{s}=1}^{K_{s}} p_{j_{s}}^{s}(t) L C_{j_{s}}(t)$

The MSS system reliability indices at sub-period $t$ can be calculated as

$$
\left\{\begin{array}{l}
L_{\mathrm{LOLP}}(t)=\sum_{s_{i}=1}^{R_{s}} \alpha_{s_{i}} L_{\mathrm{LOLP}, s_{i}}(t) \\
E_{\mathrm{EENS}}(t)=\sum_{s_{i}=1}^{R_{s}} \alpha_{s_{i}} E_{\mathrm{EENS}, s_{i}}(t) \\
R_{s}=\prod_{i=1}^{N} \prod_{L=1}^{R_{L}} \prod_{w=1}^{R_{W}} r_{i, L} r_{i, w} \\
\alpha_{s_{i}}=\prod_{i=1}^{N} \alpha_{i, L} \alpha_{i, w}
\end{array}\right.
$$

where $R_{s}, R_{L}, R_{W}$ are total numbers of the combination of system pattern, the load pattern and the wind farm output pattern, respectively; $\alpha_{s_{i}}, \alpha_{i, L}, \alpha_{i, w}$ are the probabilities of system pattern $r_{s_{i}}$, the pattern of the $i$-th load $r_{i, L}$, and the wind power output pattern of the $i$-th wind farm $r_{i, w}$, respectively.

We can obtain reliability indices, such as Loss of Load Expectation $L_{L O L E}$ and Expected Energy Not Supplied $E_{E E N S}$ over different periods through accumulation based on the dynamic probability distribution of the conventional generators and different patterns of wind farm or loads:

$$
\left\{\begin{array}{l}
L_{\mathrm{LOLE}}=\sum_{t=1}^{T} L_{\mathrm{LOLP}}(t) T^{\prime} \\
E_{\mathrm{EENS}}=\sum_{t=1}^{T} E_{\mathrm{EENS}}(t)
\end{array}\right.
$$

where $T^{\prime}$ is the number of hours for each sub-period, and $T^{\prime}=1$ in this paper, $T$ is the total number of sub-periods for reliability assessment.

\section{Case study}

The IEEE-RTS has been modified to illustrate the proposed models and techniques: original data can be found elsewhere [18]. Table 1 shows the minimum output of different types of units.

The IEEE-RTS load is a scaled-down version of load shape from the power grid of Shandong province with a 2,850 MW peak load. The load data of the test system are constructed by using the load data of power grid of Shandong province in 2009 with a 39,025 MW peak load and the scaling factor of $2,850 / 39,025$. The study period is one year. We make use of the several years' historical hourly wind speed data of Zhangbei wind farm in North China with a total wind capacity of $680 \mathrm{MW}$.

\subsection{Clustering of wind power and UGF model}

To balance efficiency and accuracy, choosing a suitable number of clusters is important. Guidelines on choosing a

Table 1 Minimum output of conventional units

\begin{tabular}{lclc}
\hline $\begin{array}{l}\text { Generation } \\
\text { type }\end{array}$ & $\begin{array}{l}\text { Minimum output } \\
\text { (MW) }\end{array}$ & $\begin{array}{l}\text { Generation } \\
\text { type }\end{array}$ & $\begin{array}{l}\text { Minimum output } \\
\text { (MW) }\end{array}$ \\
\hline U12 & 0.0 & $\mathrm{U} 155$ & 86.8 \\
U20 & 0.0 & $\mathrm{U} 197$ & 0.0 \\
U50 & 0.0 & $\mathrm{U} 350$ & 192.5 \\
U76 & 41.0 & $\mathrm{U} 400$ & 400.0 \\
U100 & 0.0 & & \\
\hline
\end{tabular}




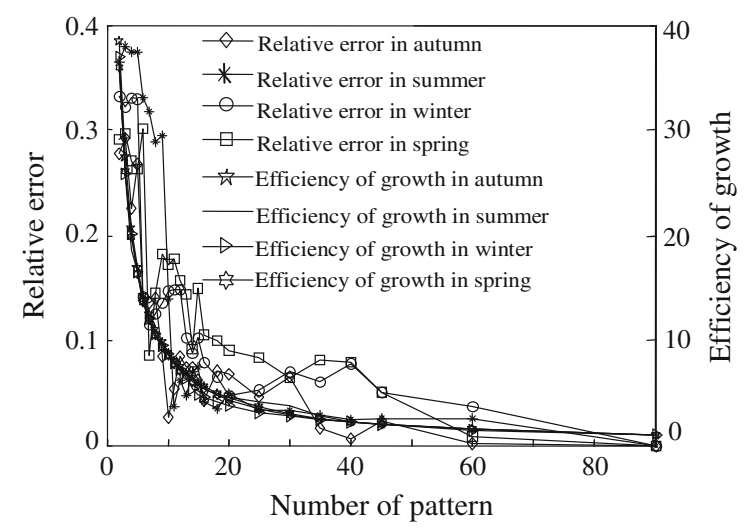

Fig. 2 Relationship between the number of clusters and the relative errors or growth efficiency

Table 2 Probability of each pattern

\begin{tabular}{llllll}
\hline Season & \multicolumn{2}{l}{ Probability $(\%)$} & $\begin{array}{l}\text { Pattern coverage } \\
\text { rate }(\%)\end{array}$ \\
\cline { 2 - 5 } & $\begin{array}{l}\text { Pattern } \\
1\end{array}$ & $\begin{array}{l}\text { Pattern } \\
2\end{array}$ & $\begin{array}{l}\text { Pattern } \\
3\end{array}$ & $\begin{array}{l}\text { Pattern } \\
4\end{array}$ & \\
\hline Spring & 27.78 & 8.89 & 38.89 & 8.89 & 84.45 \\
Summer & 49.00 & 22.22 & 17.78 & - & 89.00 \\
Autumn & 33.33 & 30.00 & 16.67 & - & 80.00 \\
Winter & 26.67 & 42.22 & 21.11 & - & 90.00 \\
\hline
\end{tabular}

suitable number of clusters are obtained based on statistical data in this case. Relative errors and growth efficiency are calculated over different seasons and the results are shown in Fig. 2.
Figure 2 indicates that the relationship between the number of clusters and the relative error is non-linear. The relationship between the number of clusters and the growth efficiency is non-linear. The expected number of the clusters would be found according to the inflexion value in the curve. Considering the relative error and growth efficiency, the number of clusters is chosen between $2 \%$ and $5 \%$ of the number of total research days, thus the number of clusters is 48 per annum.

Given space limitations, only the main modes with larger probabilities are listed. The centroid of each cluster and its corresponding probability are shown in Table 2 and Fig. 3.

As can be seen, the fluctuations and the corresponding probability of different clusters varied, the method can readily extract the salient and time-varying patterns of wind generation. Moreover, the characteristics varied seasonally: the low average power pattern has a larger probability of $49 \%$ in Summer, however, the high average power patterns has the larger probabilities of $42.22 \%$ and $38.89 \%$, respectively in Winter and Spring, showing that the proposed method can extract the seasonal characteristics of wind power output.

Four to six states can reflect the characteristics of wind speed and load [19], so we can choose four-state models for wind farms in each sub-period by an apportioning method. Multi-state models of wind power output are only shown in sub-periods 4 to 8 , and 16 to 20 of the pattern 2 in Summer in Table 3 due to space limitations.

As can be seen, the value and the probability of wind power output in different sub-periods varied, hence, the
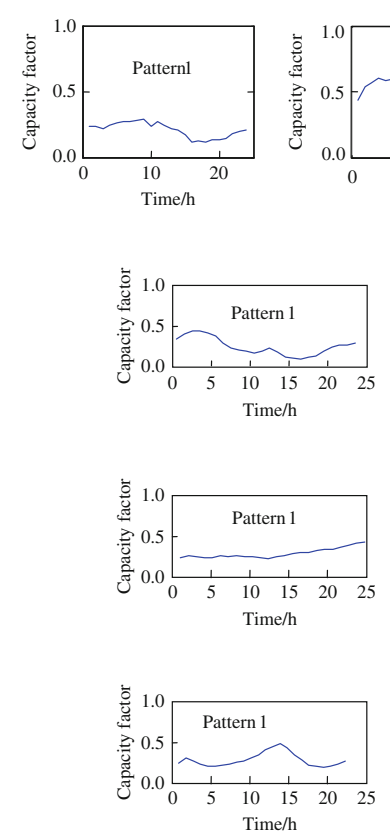$$
\text { (n) }
$$

Fig. 3 Centroids of wind power patterns in a year
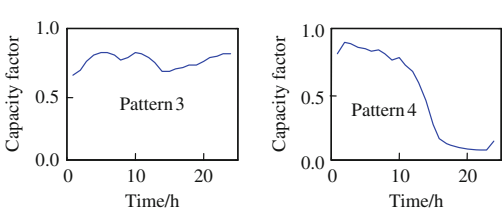

(a) Spring
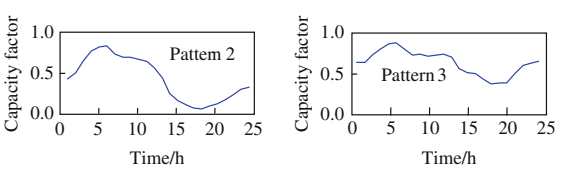

(b) Summer
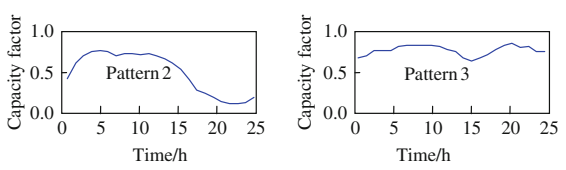

(c) Autumn

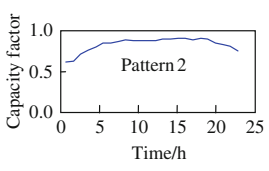

(d) Winter

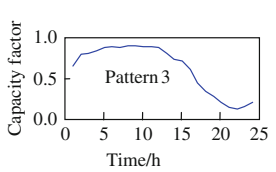

(n) 
Table 3 Wind power (WP) and probability ( $p$ ) in UGF model

\begin{tabular}{|c|c|c|c|c|c|c|c|}
\hline Period & $\begin{array}{l}\text { WP } \\
(\mathrm{MW})\end{array}$ & $\begin{array}{l}\mathrm{WP} / \\
\Delta c\end{array}$ & $p$ & Period & $\begin{array}{l}\text { WP } \\
(\mathrm{MW})\end{array}$ & $\begin{array}{l}\mathrm{WP} / \\
\Delta c\end{array}$ & $p$ \\
\hline \multirow[t]{4}{*}{4} & 120 & 12 & 0.111 & 16 & 80 & 8 & 0.296 \\
\hline & 264 & 26 & 0.148 & & 211 & 21 & 0.222 \\
\hline & 409 & 41 & 0.296 & & 342 & 34 & 0.296 \\
\hline & 553 & 55 & 0.444 & & 472 & 47 & 0.185 \\
\hline \multirow[t]{4}{*}{5} & 181 & 18 & 0.111 & 17 & 57 & 6 & 0.296 \\
\hline & 302 & 30 & 0.148 & & 168 & 17 & 0.296 \\
\hline & 422 & 42 & 0.296 & & 279 & 28 & 0.148 \\
\hline & 542 & 54 & 0.444 & & 390 & 39 & 0.259 \\
\hline \multirow[t]{4}{*}{6} & 285 & 28 & 0.222 & 18 & 61 & 6 & 0.370 \\
\hline & 372 & 37 & 0.148 & & 173 & 17 & 0.259 \\
\hline & 458 & 46 & 0.259 & & 285 & 29 & 0.185 \\
\hline & 545 & 55 & 0.370 & & 397 & 40 & 0.185 \\
\hline \multirow[t]{4}{*}{7} & 269 & 27 & 0.333 & 19 & 69 & 7 & 0.407 \\
\hline & 360 & 36 & 0.074 & & 176 & 18 & 0.296 \\
\hline & 451 & 45 & 0.296 & & 283 & 28 & 0.222 \\
\hline & 543 & 54 & 0.259 & & 390 & 39 & 0.074 \\
\hline \multirow[t]{4}{*}{8} & 225 & 23 & 0.185 & 20 & 60 & 6 & 0.518 \\
\hline & 339 & 34 & 0.222 & & 179 & 18 & 0.296 \\
\hline & 454 & 45 & 0.333 & & 298 & 30 & 0.111 \\
\hline & 568 & 57 & 0.259 & & 417 & 42 & 0.074 \\
\hline
\end{tabular}

Table 4 Observed numbers $k_{j_{m} q_{m}}$ of the unit transiting from state $j_{m}$ to state $q_{m}$, generating capacity $P_{j_{m}}$ and residence accumulated time $T_{\sum j_{m}}$ in state $j_{m}$

\begin{tabular}{lcccccl}
\hline $\begin{array}{l}\text { State } \\
\text { number }\end{array}$ & 1 & 2 & 3 & 4 & $\begin{array}{l}\text { Generating } \\
\text { capacity } P_{j_{m}} \\
(\mathrm{MW})\end{array}$ & \multicolumn{2}{l}{$\begin{array}{l}\text { Accumulated } \\
\text { time } T_{\sum j_{m}}(\mathrm{~h})\end{array}$} \\
\hline 1 & - & 6 & 1 & 0 & 0 & 75 \\
2 & 1 & - & 11 & 1 & 117 & 34 \\
3 & 0 & 3 & - & 37 & 233 & 104 \\
4 & 6 & 4 & 28 & - & 350 & 40,711 \\
\hline
\end{tabular}

traditional UGF method is crude. The time varying UGF can reflect the sequential volatility of wind power output. The common capacity factor $\Delta c=10 \mathrm{MW}$, all of the state values are expressed as integer multiples of $\Delta c$, and the number of states combination of MSS are prevented from growing exponentially.

\subsection{Time dependent state probability of the conventional generator}

A typical real coal-fired generating unit U350 is observed over 5 years [14]. The observed numbers $k_{j_{m} q_{m}}$ of the unit transition from state $j_{m}$ to state $q_{m}$ are presented in Table 4 . The generator expectation output $P_{j_{m}}$ and residence accumulated time $T_{\sum j_{m}}$ for each state $j_{m}$ are also shown.
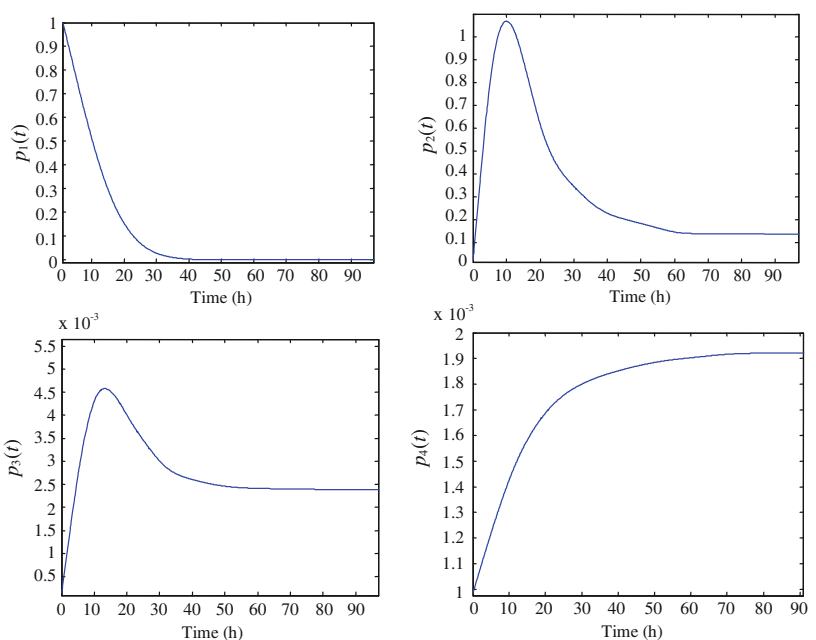

Fig. 4 Time dependent state probability of U350

Table 5 Simulation results: seasonal variations

\begin{tabular}{llcll}
\hline Season & $\begin{array}{l}L_{\text {LOLE }}(\mathrm{h} / \\
\text { a) }\end{array}$ & $E_{\text {EENS }}(\mathrm{MWh})$ & $\begin{array}{l}\text { Fuel cost } \\
\left(1 \times 10^{7} \mathrm{USD}\right)\end{array}$ & $\begin{array}{l}\mathrm{CO}_{2} \\
\text { emissions } \\
\left(1 \times 10^{5} \text { ton }\right)\end{array}$ \\
\hline Spring & 0.28 & 2.5489 & 1.3767 & 3.9966 \\
Summer & 1.86 & 199.65 & 2.9598 & 5.1262 \\
Autumn & 0.55 & 35.959 & 2.1897 & 4.8230 \\
Winter & 1.14 & 109.64 & 2.4521 & 4.9956 \\
Year & 3.83 & 347.7979 & 8.9783 & 18.9414 \\
\hline
\end{tabular}

By using (10) to (12), the following matrix of point estimations of transition intensities is computed:

$$
\left|a_{j_{m} q_{m}}\right|=\left|\begin{array}{cccc}
0.9067 & 0.0800 & 0.0133 & 0 \\
0.0294 & 0.6177 & 0.3235 & 0.0294 \\
0 & 0.0288 & 0.6154 & 0.3558 \\
0.0002 & 0.0001 & 0.00070 & 0.999
\end{array}\right|
$$

Each $a_{j_{m} q_{m}}$ in (19) is represented by such units as $1 / \mathrm{h}$.

Therefore, we can obtain the time dependent state probability of the typical conventional generator U350 with $L z$-transform. The state probabilities are changed with time in initial state, as shown in Fig. 4.

From these figures, it is observed that the state probability of U350 is time-varying, and the difference is great enough, therefore the unit steady-state (long-term) reliability cannot characterize the transient (short-term) reliability.

\subsection{Probabilistic production simulation: seasonal variations}

We divided the wind power data into four classes according to season, and then acquired the wind power pattern in each season. The results are shown in Table 5. 
Table 6 Simulation results: Summer patterns

\begin{tabular}{lllll}
\hline Load & $\begin{array}{l}\text { Wind power } \\
\text { output (\%) }\end{array}$ & $\begin{array}{l}E_{\text {EENS }} \\
(\mathrm{MWh})\end{array}$ & $\begin{array}{l}\text { Fuel costs } \\
\left(1 \times 10^{5}\right. \\
\text { USD) }\end{array}$ & $\begin{array}{l}\mathrm{CO}_{2} \\
\text { emissions } \\
\text { (kton) }\end{array}$ \\
\hline $\begin{array}{c}\text { L1 Workday } \\
\text { (5/7) }\end{array}$ & $49(\mathrm{R} 1)$ & 0.1523 & 1.2936 & 4.3693 \\
& $22.22(\mathrm{R} 2)$ & 0.0556 & 1.0500 & 3.8962 \\
L2 Weekday & $49(\mathrm{R} 1)$ & 0.3578 & 0.6521 & 2.5901 \\
$(2 / 7)$ & $22.22(\mathrm{R} 2)$ & 0.3488 & 0.5168 & 2.1102 \\
& $17.78(\mathrm{R} 3)$ & 0.0135 & 0.4001 & 1.6809 \\
\hline
\end{tabular}

Note: L1 denotes load pattern 1, L2 denotes load pattern 2; R1, R2, and $\mathrm{R} 3$ are wind power output patterns 1,2 , and 3, respectively

We can see that the reliability indices in different seasons varied. The reliability index in spring is the lowest, because the probability of high wind power patterns is larger and the load is lower. The reliability index in summer is the largest, because the probability of low wind power patterns is larger and the load is higher. In addition, fuel cost and $\mathrm{CO}_{2}$ emissions in summer are larger, because the low wind power patterns widen the peaks and valleys of the equivalent system load, and need more peaking units. The method can reflect the correlation of wind power and load: this correlation is seasonal.

\subsection{Probabilistic production simulation considering wind power and load pattern}

Take summer as an example: the time-varying wind power pattern is shown in Fig. 3, and then the UGF model for time-varying wind power output is established. The workday pattern and weekday pattern of load according to the weekly load characteristics are acquired, and the probabilistic production simulation is done and the simulation results, such as the reliability index, fuel costs, and $\mathrm{CO}_{2}$ emissions are shown in Table 6 .

In the same load pattern, the reliability index is different for different wind power patterns. Furthermore, in the same wind power pattern, the reliability index, fuel cost, and $\mathrm{CO}_{2}$ emissions vary with different load patterns due to the differences in the correction between wind power and load. In a further illustration, the impact of wind power on power systems is not only related to the wind power pattern itself, but also the correction between the wind power and the load.

\section{Conclusions}

An improved universal generating function based on probabilistic production simulation with wind power penetration is proposed. Meanwhile, by clustering the wind power data from the Zhangbei wind power farm in North of China, the IEEE-RTS is modified to test the probabilistic production simulation. The conclusions are summarised as follows:

1) The salient, time-varying pattern of wind power has been extracted by feature extraction and cluster technology.

2) A sequential UGF model is established for conventional generators with discrete-states continuous-time Markov process. By using the model, reliability indices can be more accuracy by considering specific load and wind farm output patterns of system.

3) The improved UGF method can effectively calculate the system reliability over different time and specific wind power output patterns.

4) The proposed approach explicitly quantifies the impact of the temporal correlation between wind power and system load on reliability indices, fuel cost, and $\mathrm{CO}_{2}$ emissions.

Acknowledgements This work was supported by the National High Technology Research and Development Program of China (863 Program) (No. 2011AA05A101), and National Natural Science Foundation of China (No. 51177092).

Open Access This article is distributed under the terms of the Creative Commons Attribution License which permits any use, distribution, and reproduction in any medium, provided the original author(s) and the source are credited.

\section{References}

[1] Billinton R, Chen H, Ghajar R (1996) A sequential simulation technique for adequacy evaluation of generating systems including wind energy. IEEE Trans Energy Conver 11(4):728-734

[2] Billinton R, Bai G (2004) Generating capacity adequacy associated with wind energy. IEEE Trans Energy Conver 19(3): 641-646

[3] González-Fernández RA, Leite da Silva AM (2011) Reliability assessment of time dependent systems via sequential cross entropy Monte Carlo simulation. IEEE Trans Power Syst 26(4):2381-2389

[4] Vallée F, Brunieau G, Pirlot M et al (2011) Optimal wind clustering methodology for adequacy evaluation in system generation studies using nonsequential Monte Carlo simulation. IEEE Trans Power Syst 26(4):2173-2184

[5] Wang X, Dai H, Thomas RJ (1984) Reliability modeling of large wind farms and associated electric utility interface systems. IEEE Trans Power Appar and Syst 103(3):569-575

[6] Zhang Jietan, Chen Haozhong, Hu Zechun et al (2009) Power system probabilistic simulation including wind farms. Proc CSEE 29(28):34-39 (in Chinese)

[7] Chen Shuyong, Dai Nianzhu, Bai Xiaomin et al (2000) Power system probabilistic simulation including wind farms. Electric Power 33(3):30-32 (in Chinese)

[8] Zou Bin, Li Dong (2012) Power system probabilistic production simulation with wind generation based on available capacity distribution. Proc CSEE 32(7):23-31 (in Chinese) 
[9] Ding Y, Wang P, Goel L et al (2011) Long-term reserve expansion of power systems with high wind power penetration using universal generating function methods. IEEE Trans Power Syst 26(2):766-774

[10] Levitin $G$ (2005) The universal generating function in reliability analysis and optimization. New York, Springer, pp 6-11

[11] Han Jiawei, Kamber M (2007) Data mining concepts and techniques. China Machine Press, Beijing, 51p

[12] Gomez-Munoz VM, Porta-Gandara MA (2002) Local wind patterns for modeling renewable energy systems by means of cluster analysis techniques. Renewable Energy 25(2):171-182

[13] Mehrtash A, Wang P, Goel L (2010) Reliability evaluation of restructured power systems with wind farms using equivalent multi-state models. In: International Conference on Probabilistic Methods Applied to Power Systems, Singapore, Singapore, IEEE, 2010, pp 716-721

[14] Lisnianski A, Elmakias D et al (2012) A multi-state Markov model for a short-term reliability analysis of a power generating unit. Reliab Eng Sys Safe 98(1):1-6

[15] Lisnianski A, Llia F (2012) Recent advances in system reliability. New York, Springer 2012:79-95

[16] Chen P, Pedersen T, Back-Jensen B et al (2010) ARIMA-based time series model of stochastic wind power generation. IEEE Trans Power Syst 25(2):667-676

[17] Kang Chongqing, Xia Qing, Xiang Niande (2002) Sequence operation theory and its application in power system reliability evaluation. Reliab Eng Syst Safe 78(2):101-109

[18] Probabilistic Methods Subcommittee (1979) IEEE reliability test system. IEEE Trans Power Appar and Syst 98(6):2047-2054
[19] Billinton R, Gao Y (2008) Multistate wind energy conversion system models for adequacy assessment of generating systems incorporating wind energy. IEEE Trans Energy Conver 23(1): $163-170$

\section{Author Biographies}

Hongtao WANG received the Ph.D. degree in 2005 from the School of Electrical Engineering of Shandong University, China. He is now a professor in the School of Electrical Engineering of Shandong University. His research interests include power system stability analysis, power system dispatching with intermittency and renewable energy, and power system restoration.

Xu LIU received the Master degree in 2013 from the School of Electrical Engineering of Shandong University, China. She is now a engineer of State Grid Zhejiang Electric Power Company Jiaxing Electric Power Supply Company. Her research interests include power system planning with renewable energy and power system probabilistic cost production simulation with intermittency and renewable energy.

Chunyi WANG received the Ph.D. degree in 2010 from the School of Electrical Engineering in Shandong University, China. He is now an senior engineer of State Grid Shandong Electric Power Company. His research interests include power system reliability analysis and power grid planning. 